\title{
Frecuencia de Toxoplasmosis en Cerdos Provenientes de Granjas Tecnificadas y el Sexo como Factor de Riesgo
}

\author{
Frequency of Toxoplasma gondii in Technologically Advanced Pig Farms \\ AND SEX AS A RISK FACTOR \\ Fernando Carranza C. ${ }^{1}$, Francisco Suárez A. ${ }^{1,3}$, Amanda Chávez V. ${ }^{2}$, Eva Casas A. ${ }^{2}$
}

\section{Resumen}

El objetivo del presente estudio fue estimar la frecuencia de cerdos provenientes de crianza tecnificada reactores a toxoplasmosis y la posible asociación del sexo con la positividad a la toxoplasmosis. Se tomaron 240 muestras de sangre de cerdos provenientes de granjas tecnificadas en un camal ubicado en la ciudad de Lima, Perú. Las muestras fueron analizadas mediante el método de inmunofluorescencia indirecta (IFI). La frecuencia de cerdos positivos a toxoplasmosis fue de $21.7 \%$. La evaluación del sexo como posible factor de riesgo arrojó un Odds Ratio (OR) de 1.24 con intervalo de confianza de 0.88-3.06. Se concluye que hay una frecuencia importante de cerdos provenientes de granjas tecnificadas positivos a toxoplasmosis y que el sexo no influye en la presencia de esta enfermedad.

Palabras clave: Toxoplasma gondii, toxoplasmosis, cerdo, inmunofluorescencia indirecta

\section{AbSTRACT}

The aim of the current study was to estimate the frequency of pigs from technologically advanced farms positive to toxoplasmosis and to evaluate the association between positive reaction and sex of the animal. Blood samples were collected in 240 animals in a slaughterhouse in Lima, Peru. Samples were analyzed by the indirect immunofluorescence assay (IFA). The frequency of pigs positive to toxoplasmosis was $21.7 \%$. Sex as possible

${ }^{1}$ Laboratorio de Epidemiología y Economía Veterinaria, ${ }^{2}$ Laboratorio de Microbiología y Parasitología Veterinaria, Facultad de Medicina Veterinaria, Universidad Nacional Mayor de San Marcos, Lima, Perú

${ }^{3}$ E-mail: francisco_suarez2001@hotmail.com

Recibido: 17 marzo de 2016

Aceptado para publicación: 2 de junio de 2016 
risk factor showed an Odds Ratio (OR) of 1.24 with confidence intervals of $0.88-3.06$. It was concluded that there is an important frequency of positive pigs to toxoplasmosis and that sex has no influence in the presentation of this disease.

Key words: Toxoplasma gondii, toxoplasmosis, pig, indirect immunofluorescence assay

\section{INTRODUCCIÓN}

Toxoplasma gondii es un parásito protozoario, del tipo intracelular obligado no flagelado, el cual pertenece a la Familia Sarcocystidae, Subclase Coccidia, siendo la única especie del género Toxoplasma (De Craeyé, 2012). El parásito es de carácter zoonótico, pues puede infectar a los humanos y animales de sangre caliente. Además, es uno de los parásitos más comunes, habiendo sido encontrado desde Alaska hasta Australia (Hill y Dubey, 2002), siendo más prevalente en países de clima tropical y subtropical; no obstante, se le considera el parásito más prevalente en la población mundial humana, afectando a dos tercios de ella (Gilot-Fromont et al., 2012).

La toxoplasmosis está relacionada con costumbres higiénicas, nivel socioeconómico, sanidad y contacto con hospederos definitivos (Díaz-Suárez et al., 2001). Los animales, incluyendo el humano, pueden infectarse mediante tres formas: por ingestión de ooquistes, por ingestión de quistes en tejidos de hospederos intermediarios y por transmisión transplacentaria de taquizoítos (Falco de Brito et al., 2002). El consumo de carne poco o mal cocinada ha sido relacionado con la seropositividad de $T$. gondii en diversos estudios en carcasas de ovinos, caprinos, porcinos, bovinos y aves (De Craeyé, 2012).

El cerdo es un animal que se infecta con T. gondii con facilidad (Dubey et al., 1996), aunque la modernización de los sistemas de producción porcina ha permitido una dismi- nución de este tipo de infecciones (Dubey, 2009). No obstante, persisten algunos factores que se relacionan con su frecuencia, tales como el acceso de animales del exterior, suero caprino en el alimento y presencia de gatos y roedores (De Craeyé, 2012).

El objetivo del presente estudio fue estimar la frecuencia de cerdos provenientes de crianza tecnificada reactores a toxoplasmosis y establecer la posible asociación del sexo del animal con la positividad a la parasitosis.

\section{Materiales y MéTodos}

\section{Lugar de Estudio}

La toma de muestras se llevó a cabo durante los meses de noviembre y diciembre de 2012 en el camal Conchucos, ubicado en el distrito de El Agustino, Lima, y el procesamiento de las muestras se realizó en los laboratorios de la Facultad de Medicina Veterinaria de la Universidad Nacional Mayor de San Marcos, Lima, Perú.

\section{Tamaño de Muestra y Animales}

Se obtuvieron 240 muestras de sangre de cerdos provenientes de granjas tecnificadas (120 machos y 120 hembras). El tamaño de muestra se determinó mediante la fórmula de aproximación normal a la distribución binomial para poblaciones infinitas (Daniel, 1996), con un resultado de 237 muestras. 
Cuadro 1. Resultados de la prueba de inmunofluorescencia indirecta para identificación de Toxoplasma gondii en cerdos de granjas tecnificadas, según sexo

\begin{tabular}{lccc}
\hline \multirow{2}{*}{ Sexo } & Muestras & \multicolumn{2}{c}{ Muestras positivas } \\
\cline { 3 - 4 } & $(\mathrm{n})$ & $\%$ & $\mathrm{IC}_{(0.95)}$ \\
\hline Hembra & 120 & 25.8 & $180.0-33.7$ \\
Macho & 120 & 17.5 & $190.7-24.3$ \\
\hline Total & 240 & 21.7 & $16.5-26.9$ \\
\hline
\end{tabular}

\section{Manejo de Muestras}

Las muestras $(5 \mathrm{ml})$ fueron obtenidas usando tubos Vacutainer ${ }^{\circledR}$ sin anticoagulante en el momento del sacrificio por degüello del animal. Las muestras fueron transportadas en gradillas y en una caja térmica acondicionada con hielo y gel para su conservación hacia el laboratorio, donde se procedió a la separación del suero mediante centrifugación por 10 minutos a $900 \mathrm{~g}$.

Se utilizó la técnica diagnóstica de inmunoflourescencia indirecta (IFI), utilizando reactivos comerciales (VMRD, EEUU), tales como láminas antigenadas de $T$. gondii, conjugado anti-anticuerpo porcino IgG y fluido de montaje 50/50 (Glicerol/PBS).

La prueba consiste en el uso de una lámina que contiene taquizoítos incubados con diluciones del suero problema. Los anticuerpos del suero son revelados con un anticuerpo secundario marcado con una molécula fluorescente (isocianato de fluoresceína). Las láminas se observan en un microscopio de fluorescencia. Se consideraron como positivas las diluciones a partir de 1:16 (Cortés y Macer, 2009).

\section{Análisis de Datos}

Los resultados, tanto el estimador puntual (proporción) como los límites del intervalo de confianza, son expresados en forma porcentual, según Almeida y Rouquayrol
(1992). Se evaluó la posible asociación del sexo con la positividad a la prueba mediante el Odds Ratio (OR).

\section{Resultados}

Se encontraron 52 muestras positivas a T. gondii de las 240 analizadas, resultando en una frecuencia de $21.7 \%$ ( $16.5-26.9 \%$ ). Las frecuencias de animales positivos según el sexo se presentan en el Cuadro 1.

El análisis de asociación Odds Ratio dio un valor de 1.64 con un intervalo de confianza al 95\% de 0.88-3.06, indicando ausencia de asociación estadística entre el sexo de los cerdos y la positividad de $T$. gondii.

\section{Discusión}

El cerdo es importante en la transmisión de toxoplasmosis, al poder infectar al hombre mediante la ingestión de carne infectada e insuficientemente cocida (Dubey, 2009) o a través de la manipulación de carcasas de cerdos (Suárez et al., 1999). Por otro lado, el empleo de la prueba serológica de IFI se fundamenta en que las técnicas histopatológicas son ineficientes a la detección de $T$. gondii, puesto que hay poca concentración del parásito en la carne destinada para el consumo humano (Dubey, 2009). Además, esta técnica proporciona resultados equivalentes a los 
dados por la reacción Sabin-Feldman (Soulsby, 1987; Mejía, 2009), con la ventaja de no necesitar parásitos vivos, haciéndola más segura (Acha y Szyfres, 2003).

La ausencia de asociación entre el sexo y la positividad a $T$. gondii encontrada en el presente estudio fue reportada asimismo por Arias et al. (1994).

El $21.7 \%$ de frecuencia de animales reactores podría considerarse elevada si se considera que Dubey (2009) señala que la modernización de los sistemas de producción conduce a la disminución de la adquisición de esta infección. No obstante, en la zona de Lima parece mantenerse el nivel de animales infectados, pues la frecuencia hallada no difiere del $25.2 \%$ encontrada por Bustamante y Suárez (2000) hace 16 años en cerdos del mismo tipo de crianza y lugar. Además, otro estudio realizado por Saavedra y Ortega (2004) pocos años después ofreció un resultado similar (27.7\%), mediante la técnica de Western Blot.

En otros países se reportaron resultados similares. Por ejemplo, Millar et al. (2008) reportaron el $25.5 \%$ en Paraná, Brasil, usando la misma técnica de IFI, mientras que Amaral et al. $(1975,1978)$ encontraron 22.8 y $24.7 \%$ en Brasil usando la técnica de hemaglutinación indirecta. Asimismo, en Alemania con la técnica de ELISA se han reportado frecuencias de $20.5 \%$ (Fehlhaber et al., 2003), $18.5 \%$ (Damriyasa et al., 2004) y $9.3 \%$ (Damriyasa y Bauer, 2005) trabajando entre 400 y 1500 muestras. Por otro lado, frecuencias de toxoplasmosis porcina en cerdos es usualmente más baja. Así, Kijlstra et al. (2004, 2008) reportaron $2.9 \%$ usando las técnicas de ELISA e inmunoflourescencia indirecta y $10.9 \%$ mediante la técnica de LAT (prueba de aglutinación en latex).

Frecuencias mayores de toxoplasmosis porcina han sido reportadas en Costa Rica (Arias et al., 1994), 43.8\% utilizando la técnica de IFI, aunque se utilizó un punto de corte de 1/20 con 496 muestras. En Chile se encontró $28.1 \%$ de muestras positivas a $T$. gondii (Tamayo et al., 1990) mediante la técnica de Sabin-Feldman, donde se utilizó un punto de corte de 1/16. En Panamá, Correa et al. (2008) encontraron una prevalencia de $32.1 \%$ utilizando la técnica de IFI con $1 / 20$ como punto de corte, en tanto que en Uruguay encontraron una frecuencia de $70.2 \%$ mediante la técnica de Sabin-Feldman con un punto de corte de 1/16 en 601 muestras (Freyre et al., 1991).

Las diferencias observadas en los diferentes estudios pueden atribuirse a factores metodológicos como la prueba diagnóstica utilizada, el punto de corte o variables ambientales ligadas al manejo higiénico-sanitario (Tsutsui et al., 2003). Además, en países de la Unión Europea hay un control más estricto en bioseguridad e inocuidad alimentaria (FAO, 2007).

\section{Conclusiones}

- Se detectó $21.7 \%$ de cerdos reactores a toxoplasmosis en granjas porcinas tecnificadas, constituyendo un riesgo potencial a la salud humana, la cual podría ser transmitida principalmente por el consumo de carne inadecuadamente cocida.

- No se encontró relación significativa de toxoplasmosis porcina en relación al sexo mediante la prueba de Odds Ratio.

\section{Literatura Citada}

1. Acha P, Scyfres B. 2003. Zoonosis y enfermedades parasitarias transmisibles comunes al hombre y los animales. Vol III. Parasitosis. Lima: Publicación Científica y Técnica N. ${ }^{\circ} 580$. OPS. 413 p.

2. Almeida Filho N, Rouquayrol M. 1992. Introducao a epidemiologia moderna. $2^{\circ}$ ed. Belo Horizonte, Brasil: Ed Coopmed. 184 p. 
3. Amaral V, Santos S, Bella Neto J. 1978. Levantamento sorológico da toxoplasmose suína latente em alguns municipios do Estado de São Paulo, Brasil. Biológico 44: 155-158.

4. Amaral V, Santos S, Rebouças $M$. 1975. Estudos preliminares sobre a prevalência de anticorpos provenientes dos Estados de São Paulo e Rio Grande do Sul, Brasil. Biológico 41: 105-107.

5. Arias M, Reyes L, Chinchilla M, Linder E. 1994. Seroepidemiology of Toxoplasma gondii (Apicomplexa) in meat producing animals in Costa Rica. Rev Biol Trop 42: 15-20.

6. Bustamante J, Suárez F. 2000. Estudio comparativo de frecuencias de toxoplasmosis en porcinos procedentes de crianza tecnificada y no tecnificada. Rev Inv Vet Perú 11(1): 32-39. doi: 10.15381/rivep.v11i1.6782

7. Correa R, Cedeño L, de Escobar C, Fuentes I. 2008. Increased urban seroprevalence of Toxoplasma gondii infecting swine in Panama. Vet Parasitol 153: 9-11. doi: 10.1016/j.vetpar. 2008.01.017

8. Cortés L, Mancera L. 2009. Concordancia entre ELISA e IFI para la determinación de anticuerpos tipo IgG contra Toxoplasma gondii. Infectio 13: 76-82. doi: 10.1016/S0123-9392(09)70728-3

9. Damriyasa I, Bauer C. 2005. Seroprevalence of Toxoplasma gondii infection in sows in Munsterland, Germany. Deut Tierarztl Woch 112: 223224.

10. Damriyasa I, Bauer C, Edelhofer R, Failing K, Lind P, Petersen E, Schares $G$, et al. 2004. Cross-sectional survey in pig breeding farms in Hesse, Germany: seroprevalence and risk factors of infections with Toxoplasma gondii, Sarcocystis spp and Neospora caninum in sows. Vet Parasitol 126: 271-286.

11. Daniel W. 1996. Bioestadística. Base para el análisis de las ciencias de la salud. $5^{\circ}$ ed. México: Uteha Noriega. 905 p.
12. De Craeyé S. 2012. Toxoplasma gondii, a successful and underestimated foodborne parasite. Development of detection methods and their use for the screening of animal reservoirs. PhD Thesis. Belgium: Ghent University. $188 \mathrm{p}$.

13. Díaz-Suárez O, Parra A, AraujoFernández M. 2001. Seroepidemiología de la toxoplasmosis en una comunidad marginal del Municipio Maracaibo, Estado Zulia, Venezuela. Invest Clín 42: 107-122.

14. Dubey J. 2009. Toxoplasmosis in pigs. The last 20 years. Vet Parasitol 164: 89103. doi: 10.1016/j.vetpar.2009.05.018

15. Dubey J, Lunney J, Shen S, Kwok O, Ashford D, Thulliez P. 1996. Infectivity of low numbers of Toxoplasma gondii oocysts to pigs. J Parasitol 82: 438-443.

16. Falco de Brito A, de Souza L, da Silva A, Langoni H. 2002. Epidemiological and serological aspects in canine toxoplasmosis in animals with nervous symptoms. Mem Inst Oswaldo Cruz 97: 31-35. doi: 10.1590/S007402762002000100003

17. [FAO] Organización de las Naciones Unidas para la Agricultura y la Alimentación. 2007. Instrumentos de la FAO sobre la bioseguridad. Roma: FAO. $148 \mathrm{p}$.

18. Fehlhaber K, Hintersdorf P, Kruger G. 2003. Pravalenz von Toxoplasma gondii. Untersushugen bei Schlachtschweinen aus verschiedenen Haltungsformen und in handelsublichen Hackfleischproben. Fleishchwirtschaft 83: 97-99.

19. Freyre A, Colombo A, D'Angelo J, Falcón J. 1991. Prevalencia de la infección toxoplásmica en cerdos de Uruguay y su significación zoonótica. Avances Cienc Vet 6: 166-171. doi: 10.5354/ 0719-5273.1991.4653

20. Gilot-Fromont E, Lélu M, Dardé M, Richomme C, Aubert D, Afonso E, Mercier A, et al. 2012. The life cycle of Toxoplasma gondii in the natural environment. Intech [Internet]. Available 
in: http://cdn.intechopen.com/pdfs-wm/ 38939.pdf

21. Hill D, Dubey J. 2002. Toxoplasma gondii: transmission, diagnosis and prevention. Clin Microbiol Infect 8: 634640. doi: 10.1046/j.1469-0691.2002. 00485.x

22. Kijlstra A, Eissen O, Cornelissen J, Munniksma K, Eijck I, Kortbeek T. 2004. Toxoplasma gondii infection in animal-friendly pig production systems. Invest Ophthalmol Vis Sci 45:3165-3169.

23. Kijlstra A, Meerburg B, Cornelissen $J$, De Craeye S, Vereijken P, Jongert E. 2008. The role of rodents and shrews in the transmission of Toxoplasma gondii to pigs. Vet Parasitol 156: 183190. doi: 10.1016/j.vetpar.2008.05.030

24. Mejía T. 2009. Estudio epidemiológico retrospectivo y prospectivo de la incidencia de toxoplasmosis como enfermedad zoonótica, en mujeres, en tres hospitales de la República de El Salvador. Tesis de pregrado. El Salvador: Univ de El Salvador. $87 \mathrm{p}$.

25. Millar P, Daguer H, Vicente R, da Costa T, Sobreiro L, Amendoeira M. 2008. Toxoplasma gondii: epidemiological study of pigs from southestern
Paraná, Brazil. Pesq Vet Bras 28: 1518. doi: 10.1590/S0100-736X2008000100002

26. Saavedra G, Ortega Y. 2004. Seroprevalence of Toxoplasma gondii in swine from slaughterhouses in Lima, Peru, and Georgia, U.S.A. J Parasitol 90: 902-904.

27. Soulsby E. 1987. Parasitología y enfermedades parasitarias en los animales domésticos. $7^{\circ}$ ed. México DF: Interamericana. $823 \mathrm{p}$.

28. Suárez F, Andrade H, Galisteo A. 1999. Evaluación serológica del Toxoplasma gondii en suinos mediante prueba de ELISA. XXI Reunión Científica Asociación Peruana de Producción Animal. Puno, Perú.

29. Tamayo R, Contreras M, Méndez M, Castro M. 1990. Toxoplasmosis en cerdos beneficiados en las plantas faenadoras de Temuco y Valdivia, Chile. Arch Med Vet 22: 95-99.

30. Tsutsui V, Navarro I, Freire R, Freitas J, Prudencio L, Delbem A, Marana E. 2003. Soroepidemiologia e fatores associados à transmissão do Toxoplasma gondii em suínos do Norte do Paraná. Arch Vet Sci 8: 27- 34. 